\title{
La participación de las familias en los procesos de innovación educativa de los centros escolares
}

\author{
Maria Antònia Riera-Jaume \\ Maria Ferrer-Ribot \\ Carme Pinya-Medina \\ Bartomeu Mut-Amengual \\ Universitat de les Illes Balears. España. \\ maria.riera@uib.es \\ maria.ferrer-ribot@uib.es \\ carme.pinya@uib.es \\ tomeu.mut@uib.es
}

Recibido: 9/2/2021

Aceptado: 5/7/2021

Publicado: 31/1/2022

\section{Resumen}

El éxito de un proceso de innovación educativa depende en buena parte de que toda la comunidad pedagógica se sienta parte integrante del cambio, y a menudo las familias participan poco en estos procesos. En el presente artículo intentaremos conocer cómo es su intervención, además de su nivel de colaboración y de conocimiento en referencia a la innovación. En la investigación se ha optado por una metodología cuantitativa a través de la aplicación de un cuestionario administrado a padres y madres de diversos centros de educación infantil. Los resultados se muestran clasificados en tres categorías (conocimiento, participación y valoración) y destacan que las familias manifiestan tener un conocimiento elevado de la metodología y una valoración positiva respecto a la innovación. Las conclusiones señalan que, en relación con la metodología, todavía se desconocen aspectos concretos sobre sus beneficios a largo plazo, datos que contrastan con las percepciones parentales. A pesar de que estas metodologías son cada vez más extendidas, se debe señalar que, sobre todo, el reto está en cómo hacer efectiva una mayor participación de las familias para reforzar los efectos positivos de los cambios implementados, así como la consolidación de estas intervenciones en los centros.

Palabras clave: innovación educativa; participación familiar; metodología; ambientes de aprendizaje; educación infantil

\section{Resum. La participació de les famílies en els processos d'innovació dels centres escolars}

L'èxit d'un procés d'innovació educativa depèn en bona part del fet que tota la comunitat pedagògica se senti part integrant del canvi, i sovint les famílies participen poc en aquests processos. En el present article intentarem conèixer com hi intervenen, en quin nivell hi col-laboren i quins coneixements tenen en referencia a la innovació. A la investigació que us mostrem s'ha optat per una metodologia quantitativa a través de l'aplicació d’un qüestionari administrat a pares i mares de diversos centres d'educació infantil. Els resultats es mostren classificats en tres categories (coneixement, participació i valoració) i destaquen que les famílies manifesten que tenen un coneixement elevat de la metodologia i que 
valoren la innovació de manera positiva. Les conclusions assenyalen que, en relació amb la metodologia, encara es desconeixen aspectes concrets sobre els beneficis que mostren a llarg termini, dades que contrasten amb les percepcions parentals. Tot i que aquestes metodologies són cada vegada més esteses, cal assenyalar que, sobretot, el repte rau en la manera com es pot fer efectiva una participació més gran de les famílies per reforçar els efectes positius dels canvis implementats, així com la consolidació d'aquestes intervencions als centres.

Paraules clau: innovació educativa; participació familiar; metodologia; ambients d'aprenentatge; educació infantil

\title{
Abstract. Family participation in school educational innovation processes
}

The success of an educational innovation process depends to a large extent on the entire educational community feeling like an integral part of the change. However, families often participate little in these processes. This article aims to determine how they intervene, as well as their level of participation and knowledge in relation to innovation. The research method is quantitative and uses a questionnaire administered to families from various early childhood education centers. The results are classified into three categories: knowledge, participation, and assessment. The findings highlight that the families state that they have a high knowledge of the methodology and a positive assessment regarding innovation. The conclusions indicate that, in relation to the methodology, specific aspects of its long-term benefits are still unknown, data that contrast with the perceptions of families. Although these methodologies are increasingly widespread, it should be noted that, above all, the challenge lies in how to engage families more effectively in processes of innovation to reinforce the positive effects of the changes implemented, as well as the consolidation of these interventions in the centers.

Keywords: educational innovation; family school relationship; methodology; experimental learning; early childhood education

\author{
Sumario \\ 1. Introducción 4. Resultados \\ 2. Estado del arte 5. Discusión y conclusiones \\ 3. Metodología Referencias bibliográficas
}

\section{Introducción}

Desde hace varios años, los centros docentes están inmersos en procesos de innovación con cambios metodológicos que permitan transformar la práctica educativa alejándose de perspectivas más lineales y con un marcado carácter transmisor. El estudio que se presenta analiza el papel que tienen las familias en esta acción renovadora. Para ello se desarrolló una investigación llevada a cabo en cinco centros que implementaron modificaciones e innovaciones en la etapa de educación infantil, y la pregunta planteada fue para averiguar qué lugar ocupan las familias en los procesos de cambio y transformación educativa. 
La literatura recoge y reconoce la mejora del conjunto de la escuela con la implicación de toda la comunidad educativa como uno de los factores que condicionan no solo el éxito de la innovación, sino también la sostenibilidad de la misma (Fullan, 2007; Ramírez, 2020: Stoll y Fink, 1999; Zabalza, 2000), por ello es indispensable incorporar a las familias en este proceso de transformación, como parte activa, implicada y colaboradora del mismo. En este sentido, la presente investigación contribuye a evidenciar, una vez más, que el rol parental es clave en procesos de innovación docente.

El principal objetivo de la investigación es analizar el nivel de conocimiento, la implicación y la valoración de las madres y de los padres sobre los procesos de innovación educativa. En este caso la innovación consiste en la implementación del trabajo por ambientes de aprendizaje en la etapa de educación infantil. En los últimos años se han ido generando experiencias centradas inicialmente en una nueva concepción del espacio ambiente que conlleva cambios educativos importantes. La propuesta del trabajo por ambientes implica un replanteamiento del proyecto de centro, de manera que la escuela se va transformando en un sistema más abierto, flexible y dinámico (Riera, Ferrer y Ribas, 2014).

Según Murillo y Krichesky (2012), en una acción escolar orientada hacia el cambio lo que promueve el éxito del proceso es favorecer una cultura general de mejora; impulsar espacios de formación continua para el profesorado; distribuir roles y funciones de liderazgo, y contar con el apoyo externo y la participación de las familias y de los alumnos. Para que un proceso de mejora se desarrolle satisfactoriamente es necesario que la voluntad de cambio se haga patente e impregne a gran parte de los integrantes de un centro. Pero no se trata solo de que la comunidad sienta que es necesario hacer algo, sino también, y quizá lo más importante, que crea que efectivamente se puede cambiar.

\section{Estado del arte}

El proceso de desarrollo de una innovación pedagógica puede configurarse desde una mirada eminentemente técnica, en que el proceso sea construido jerárquicamente con grupos expertos y de poder, hasta una aproximación práctica, en que se genere desde las bases, desde los actores mismos insertos en la situación educativa (Grundy, 1997). En este camino la participación de las familias influirá en el resultado de la implementación, ya que cuantos más sectores implicados haya, más posibilidades de consolidación del proyecto existirán. Según un estudio realizado por Marcelo, Mayor y Gallego (2010), un $33,4 \%$ de los proyectos de innovación han contado con la participación de los padres y las madres, cifra que varía en función del nivel educativo en el que nos encontremos. Así, en infantil y primaria la participación parental es mucho mayor (un 42,5\%) que en secundaria (un 23,6\%).

Son muchos los autores que señalan cómo la capacidad de transformación del contexto educativo se potencia a medida que se ve incrementada la presencia de las familias, el grado de formalización y su implicación en la toma 
de decisiones (De la Guardia y Santana, 2010; Comas et al., 2014; Garreta, 2015, 2017). Estos autores destacan que los padres y las madres desean entablar un nivel profundo de relación con las escuelas, quieren participar en los contenidos educativos y entrar en las aulas. Así, manifiestan que, sintiéndose como parte afectada por las continuas y excesivas reformas curriculares y pedagógicas, se ven capacitados para debatir sobre contenidos y hacer propuestas educativas.

Una relación entre padres y profesores entendida como partners - que comparten responsabilidades como miembros de la misma comunidad educativa (Schecter y Sherry, 2009; Hoover-Dempsey, 2011) — permitiría favorecer el sentimiento de pertenencia de las familias al centro educativo (Barton et al., 2004). Sin duda, la participación, los objetivos compartidos y la comunicación fluida entre ambos son aspectos clave para afianzar las relaciones y la confianza mutua. Como señala Carbonell (2006), uno de los factores clave para promover la innovación es contar con una comunidad educativa receptiva, con una actitud abierta al cambio y con voluntad de compartir objetivos para la mejora o la transformación del centro. El estudio que aquí se presenta se centra en analizar el conocimiento, la participación y la valoración que hacen las familias en relación con el proceso de cambio e innovación. Sin duda, este puede verse favorecido en la medida en que los diversos agentes de la comunidad educativa muestran un grado de aceptación y complicidad.

Diversos estudios (Graham-Clay, 2005; Kidder, 2013; Lunts, 2003; Heath, Maghrabi y Carr, 2015; Patel, Corter y Pelletier, 2008) concluyen afirmando que el incremento de la comunicación aumenta la participación, lo que se relaciona con un mayor éxito educativo de los alumnos. Cuando los valores de la escuela y los de la familia coinciden, se desarrolla un sentimiento de pertenencia al centro que influye en los logros académicos de los estudiantes (Castro et al., 2014; Crozier y Davies, 2007; Hill y Craft, 2003; Lee y Bowen, 2006). Los efectos positivos no solo son para el niño, sino también para los profesores y para los padres (Martínez González y San Fabián, 2002).

Cambiar la forma en que las escuelas perciben la participación parental podría ayudar a avanzar hacia un mayor nivel de compromiso. Pedró (2008) señala que todavía hay maestros que se muestran reticentes a la intervención de las familias en las acciones académicas o no se sienten satisfechos con su implicación, y estas, por su parte, aunque valoran la tarea del docente, notan que no acaban de encajar o no se sienten preparadas para participar en la acción escolar, como apunta Feito (2010). También Andrés y Giró (2016) señalan que hay profesores y equipos directivos que defienden que hay espacios y ámbitos exclusivos de ellos. En este sentido, el papel del centro docente para promover que las familias participen en las iniciativas educativas es señalado en numerosos estudios (Giró y Andrés, 2016; Lewis, Kim y Bey, 2011; Vigo, Dieste y Julve, 2015).

Tal como señalan Comas, Escapa y Abellán (2014), la participación parental se puede clasificar de diversas maneras: según el tipo de acción, el nivel de institucionalización y el grado de acceso en la toma de decisiones. Para fomen- 
tar la implicación de las familias será necesario revisar también los modos y los canales de participación, tal como indica Kidder (2013), que distingue dos modalidades: las actividades y las actitudes en casa y las actividades en la escuela, siendo estas últimas las que ofrecen grandes oportunidades para fortalecer los vínculos con la comunidad educativa. El mismo autor clasifica la participación en niveles de impacto: simple, consultiva, proyectiva y metaparticipación. También Grañeras, Gil y Díaz-Caneja (2011) identifican cinco modelos de participación de las familias y de la comunidad, tal como se recoge en la tabla 1 .

Tabla 1. Modelos de participación de las familias y de la comunidad

\begin{tabular}{ll}
\hline Autores & Participación en el entorno educativo \\
\hline Comas et al. (2014) & Según el tipo de acción: colectiva o individual. \\
& $\begin{array}{l}\text { Según el nivel de institucionalización: formal o informal. } \\
\text { Según el grado de acceso en la toma de decisiones: dar y recibir } \\
\text { información, aceptar y ofrecer opiniones, consultar y hacer pro- } \\
\text { puestas, codecidir, cogestionar, etc. }\end{array}$ \\
\hline Simple: puntual, individual, informativa. \\
\hline Kidder (2013) & $\begin{array}{l}\text { Consultiva: informal, colectiva, comisiones. } \\
\text { Proyectiva: por iniciativa propia de las familias, planificada. }\end{array}$ \\
\hline Metaparticipación: decisoria, de larga duración, cogestión. \\
\hline Grañeras et al. (2011) & $\begin{array}{l}\text { Informativa. } \\
\text { Consultiva. }\end{array}$ \\
\hline Decisoria. \\
\hline Evaluativa. \\
Educativa. \\
\hline
\end{tabular}

Fuente: elaboración propia.

Epstein (2001) y Nye, Turner y Schwartz (2006) demuestran que la implicación parental incide de manera significativa en los objetivos académicos y de desarrollo de los hijos. Epstein (2001) argumenta que las escuelas que implementan prácticas que promueven asociaciones sólidas entre la escuela, la familia y la comunidad están mejor capacitadas para ayudar a los niños a tener éxito. El reto de la implementación de nuevas metodologías docentes se basaría también en incluir a la comunidad educativa.

Pérez (2012) apunta la necesidad de transformar los escenarios educativos diseñando contextos adecuados de aprendizaje, de ahí la relevancia de organizar el centro por ambientes donde puedan llevarse a cabo experiencias auténticas que generen aprendizajes significativos y relevantes, siguiendo la línea pedagógica del aprendizaje a partir de contextos experienciales (Kolb, 2014; Silberman, 2007; Smith, 2016). Es por ello que en este estudio se han escogido centros que están introduciendo cambios metodológicos, concretamente 
el trabajo por ambientes de aprendizaje. Esta propuesta nos remite al escenario educativo donde se generan condiciones favorables para el desarrollo y el aprendizaje. Supone organizar el espacio, el tiempo y los recursos de manera que se consiga un espacio rico, con grupos reducidos y heterogéneos, lo que permitirá ampliar las oportunidades de experimentación, investigación, juego y relación (Ribas, 2011). Para ello se diseñan los espacios con propuestas diversas donde se incluyen los diferentes lenguajes expresivos y los contenidos educativos de manera interrelacionada.

La finalidad de los ambientes de aprendizaje es promover que los niños sean protagonistas de su propio proceso a partir de la creación de espacios estimuladores, creativos y participativos donde los ellos actúen, pero también reflexionen sobre su propia acción desde una posición activa y competente y que posibilite la comunicación y el encuentro con las personas que habitan dicho ambiente (Riera et al., 2014). Como señala Otálora (2010, p. 80): "favorecen no sólo la adquisición de múltiples "saberes", sino que fortalecen las competencias afectivas, sociales y cognitivas necesarias para enfrentar de manera creativa las demandas crecientes del entorno durante los primeros años de vida». Sin embargo, como señala Duarte (2003), redimensionar los ambientes educativos en la escuela no solo implica modificar el espacio físico, los recursos y los materiales, sino que también exige un replanteamiento de los proyectos educativos y los modos de interaccionar de sus protagonistas, de manera que el centro docente sea un verdadero sistema abierto, flexible, dinámico y que facilite la articulación de los integrantes de la comunidad educativa (maestros, estudiantes, padres y equipo directivo).

Uno de los objetivos planteados en el estudio ha sido conocer qué información y qué posibilidades de participación tienen las familias para involucrarse en estos procesos de cambio.

\section{Metodología}

El presente trabajo es el resultado de una investigación llevada a cabo en cinco centros educativos de la comunidad autónoma de las Islas Baleares, cuatro de titularidad pública y uno de titularidad concertada. Los objetivos son analizar el nivel de conocimiento, la participación y la valoración que hacen las familias de la técnica del trabajo por ambientes. Para llevar a cabo estos objetivos se ha optado por la metodología cuantitativa a través del cuestionario como herramienta de recogida de datos.

\subsection{Muestra}

La población está formada por 693 familias y han participado en el estudio un total de 400 (un 57,72\%), de las cuales podemos indicar que:

- Un 75,8\% de los cuestionarios ha sido respondido por la madre; un 23,9\%, por el padre, y un $0,2 \%$, por el tutor legal. 
- Un 78,1\% de la muestra tiene como lugar de nacimiento las Islas Baleares; un $10,7 \%$, otras comunidades autónomas; un $10,5 \%$, otros países, y un $0,7 \%$, no responde a la pregunta.

- El nivel de estudios de la muestra es mayoritariamente superior (un $43,2 \%$ ), mientras que el $31,7 \%$ afirma tener estudios primarios, y el $24,6 \%$, secundarios; el resto (un $0,5 \%$ ) no contesta.

- Las familias que han respondido pertenecen al grupo de 3 años en un $34,7 \%$, al aula de 4 años en un 32,9\% y a la de 5 años el $32,2 \%$ restante. Existe un $0,2 \%$ que no ha respondido a esta pregunta.

- Para la mayoría de las familias que responde (un 69,7\%) se trata del primer hijo escolarizado.

\subsection{Instrumentos de recogida de datos}

Se elaboró un cuestionario ad hoc que constaba de 31 ítems, además de los datos de caracterización de la muestra. El cuestionario fue sometido en una primera fase a un proceso de contrastación a partir de un panel Delphi formado por 7 profesores universitarios, 4 maestras de educación infantil y 3 familias, todos ellos conocedores de la materia. Se obtuvo un 100\% de respuesta y un $90 \%$ de concordancia. En segundo lugar, se llevó a cabo una prueba piloto con un grupo de 20 familias, se procedió a la reformulación de dos ítems y se obtuvo una coincidencia total entre la visión del encuestador y de las familias participantes.

Se realizó el análisis de la fiabilidad de consistencia interna del instrumento mediante la prueba alfa de Cronbach para toda la escala y para cada uno de los ítems presentados en este estudio, que fue de 0,725. Estos ítems se distribuyen en tres dimensiones:

a) Conocimiento que tienen las familias respecto al proceso de implementación del trabajo por ambientes en las aulas de educación infantil.

b) Participación de los padres y/o de las madres en la metodología de trabajo por ambientes.

c) Valoración que hacen respecto a esta metodología de trabajo.

La escala de respuesta se organizó a través de una gradación Likert, donde 0 era nada, y 5 , mucho.

\subsection{Procedimiento}

Los cuestionarios fueron administrados en las cinco escuelas de referencia de las familias encuestadas. Se acompañaron de una carta de presentación y unas instrucciones de aplicación en las que se especificaban los objetivos de la investigación y el carácter anónimo de las respuestas, así como los aspectos éticos y de confidencialidad que sustentaban esta investigación. 
Para el tratamiento y los análisis de los datos recogidos se utilizó el software de tratamiento de datos estadístico SPSS (Statistical Package for the Social Sciences) en su última versión, a través de un análisis estadístico descriptivo y de frecuencia.

\section{Resultados}

De acuerdo con el diseño inicial del estudio, los resultados que se desprenden de él se exponen en tres grandes bloques y/o dimensiones según aparecen en el cuestionario: a) conocimiento, $b$ ) participación y $c$ ) valoración.

\section{a) Dimensión de conocimiento}

Entendemos por conocimiento la información de la que dispone la familia sobre la nueva metodología implementada en el centro educativo. Esta dimensión se mide en función de la información que las madres y los padres tienen de los ambientes de aprendizaje y de las estrategias que se utilizan para la búsqueda y el intercambio de información.

Tabla 2. Conocimiento sobre la implementación de la metodología por ambientes en las aulas de educación infantil

\begin{tabular}{|c|c|c|c|c|c|c|c|}
\hline & \multicolumn{5}{|c|}{ Nivel de acuerdo (\%) } & \multirow{2}{*}{ Media } & \multirow{2}{*}{$n$} \\
\hline & 1 & 2 & 3 & 4 & 5 & & \\
\hline 1. Conozco el trabajo por ambientes. & 2,3 & 9,6 & 22,7 & 47,5 & 17,9 & 3,69 & 396 \\
\hline $\begin{array}{l}\text { 2. Uno de los motivos para seleccionar } \\
\text { el centro ha sido esta manera de } \\
\text { trabajar. }\end{array}$ & 21 & 14,4 & 17,7 & 28,3 & 17,9 & 3,12 & 396 \\
\hline $\begin{array}{l}\text { 3. La información recibida ha sido sufi- } \\
\text { ciente para entender esta manera de } \\
\text { trabajar. }\end{array}$ & 1,5 & 13,1 & 28,6 & 34,9 & 21,9 & 3,63 & 398 \\
\hline $\begin{array}{l}\text { 4. He buscado información complemen- } \\
\text { taria a la aportada por la escuela. }\end{array}$ & 26,2 & 26,4 & 20,9 & 18,4 & 7,8 & 2,57 & 397 \\
\hline $\begin{array}{l}\text { 5. He comentado con otras familias la } \\
\text { conveniencia o no de esta metodo- } \\
\text { logía. }\end{array}$ & 8,5 & 14,8 & 26,1 & 33,6 & 17 & 3,36 & 399 \\
\hline $\begin{array}{l}\text { 6. Conozco qué son los diferentes } \\
\text { ambientes implementados en el } \\
\text { centro. }\end{array}$ & 3,5 & 9,3 & 19 & 41 & 27,3 & 3,79 & 400 \\
\hline $\begin{array}{l}\text { 7. Conozco la dinámica y la organiza- } \\
\text { ción de los ambientes. }\end{array}$ & 3,8 & 14,5 & 23,6 & 40,9 & 17,3 & 3,53 & 399 \\
\hline
\end{tabular}

Fuente: elaboración propia.

Cabe señalar que las familias encuestadas manifiestan tener un conocimiento elevado en lo que se refiere a este nuevo enfoque metodológico (ver tabla 2). Entre los ítems con mayor valoración encontramos al número 6 ("Conozco qué son los diferentes ambientes implementados en el centro»), con un 27,3\%; 
seguido por el ítem 3 ( «La información recibida ha sido suficiente para entender esta manera de trabajar»), con un 21,9. Por el contrario, los ítems 4 («He buscado información complementaria a la aportada por la escuela») y 2 («Uno de los motivos para seleccionar el centro ha sido esta manera de trabajar») son los que obtienen mayor índice porcentual en el nivel inferior de la escala, con un $26,2 \%$ y un $21 \%$, respectivamente, subrayando estas acciones como poco o nada.

A continuación, se planteó averiguar qué concepción tenían las familias sobre el trabajo por ambientes en cuanto a los aspectos generales de los mismos.

Tabla 3. Cómo se entiende el trabajo por ambientes (ítem 10)

\begin{tabular}{lcc}
\hline & $\boldsymbol{n}$ & $\%$ \\
\hline El alumnado cambia de aula y de maestro/a. & 99 & 24,6 \\
Cada ambiente está especializado en un área temática. & 243 & 60,4 \\
El alumnado puede escoger libremente el ambiente al que ir. & 48 & 11,9 \\
\hline Desconozco el trabajo por ambientes. & 6 & 1,5 \\
\hline
\end{tabular}

Fuente: elaboración propia.

Principalmente la organización en este nuevo planteamiento metodológico consiste en diseñar los espacios en función de áreas temáticas. Así, podemos encontrar el ambiente constructivo, el matemático, el simbólico, etc. Tal como señalan los resultados (tabla 3), las familias captan esta especialización de los espacios y entienden que esta nueva organización implica también una pérdi-

Gráfico 1. Fuente de la información sobre este tipo de trabajo que ha llegado a las familias

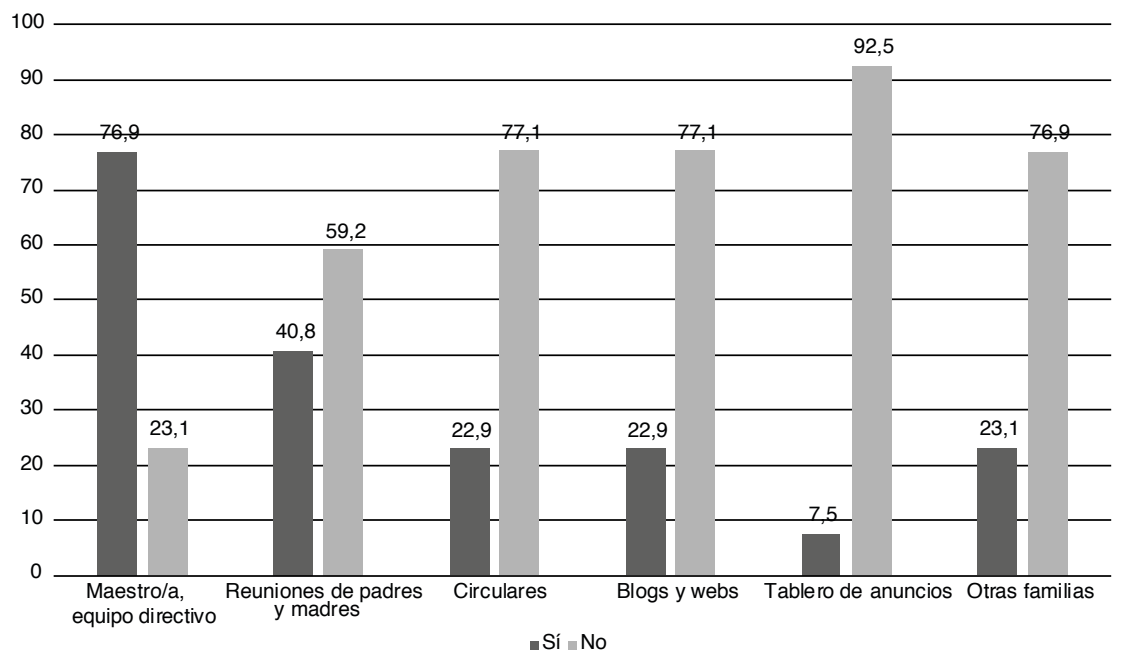

Fuente: elaboración propia. 
da de la idea de aula y permite realizar un uso compartido de los distintos ámbitos más allá de la clásica separación de los grupos por niveles.

Otro de nuestros objetivos era averiguar qué canales habían sido los más utilizados para que los centros trasladasen las informaciones a las familias. Como vemos en la siguiente gráfica, la principal fuente de información fue la que habían recibido directamente del maestro o la maestra, o del equipo directivo (un $76,9 \%$ ).

\section{b) Dimensión de participación}

En esta dimensión se quería analizar cómo era la implicación de las familias respecto a la aportación de material, la observación en las sesiones del trabajo por ambientes o la participación en comisiones para la construcción y la mejora de los espacios ambiente.

Tabla 4. Participación de las familias en la metodología del trabajo por ambientes

\begin{tabular}{|c|c|c|c|c|c|c|c|}
\hline & \multicolumn{5}{|c|}{ Nivel de acuerdo (\%) } & \multirow{2}{*}{ Media } & \multirow{2}{*}{$n$} \\
\hline & 1 & 2 & 3 & 4 & 5 & & \\
\hline $\begin{array}{l}\text { 1. Participación de forma activa en las } \\
\text { actividades del centro. }\end{array}$ & 9,2 & 24,4 & 33,8 & 24,6 & 6,2 & 2,94 & 395 \\
\hline $\begin{array}{l}\text { 2. Aportación de materiales que piden } \\
\text { los maestros y las maestras para enri- } \\
\text { quecer los ambientes. }\end{array}$ & 2 & 9,1 & 29 & 38 & 21,2 & 3,72 & 397 \\
\hline $\begin{array}{l}\text { 3. Habéis asistido en alguna ocasión } \\
\text { como observadores a las sesiones de } \\
\text { ambientes. }\end{array}$ & 65,3 & 18,3 & 11,3 & 2,5 & 2,5 & 1,59 & 398 \\
\hline $\begin{array}{l}\text { 4. Participáis de manera regular en el } \\
\text { momento del trabajo por ambientes. }\end{array}$ & 51,6 & 27,1 & 12,7 & 5,3 & 3 & 1,83 & 395 \\
\hline $\begin{array}{l}\text { 5.Participáis en comisiones de trabajo } \\
\text { en el centro para la mejora del trabajo } \\
\text { por ambientes. }\end{array}$ & 63,3 & 17,9 & 11 & 5,4 & 2,3 & 1,67 & 392 \\
\hline
\end{tabular}

Fuente: elaboración propia.

Como podemos ver, las familias participan mayoritariamente aportando materiales para enriquecer los diversos ambientes en un 38\%. Sin embargo, la participación directa de los padres es mucho menor, tal y como señalan los resultados obtenidos en los ítems 3, 4 y 5 (ver tabla 4).

\section{c) Dimensión de valoración}

En esta dimensión se pretendía recoger la valoración y el grado de satisfacción parental con el nuevo enfoque metodológico. 
Tabla 5. Valoración de la metodología del trabajo por ambientes por parte de las familias

\begin{tabular}{|c|c|c|c|c|c|c|c|}
\hline & \multicolumn{5}{|c|}{ Nivel de acuerdo (\%) } & \multirow{2}{*}{ Media } & \multirow{2}{*}{$n$} \\
\hline & 1 & 2 & 3 & 4 & 5 & & \\
\hline $\begin{array}{l}\text { 1. Consideráis interesante esta nueva } \\
\text { metodología de trabajo en el aula. }\end{array}$ & 1 & 3 & 11,6 & 33,8 & 50,6 & 4,30 & 396 \\
\hline $\begin{array}{l}\text { 2. Os gustaría que en educación prima- } \\
\text { ria se continuase con esta dinámica } \\
\text { de trabajo. }\end{array}$ & 3 & 7,1 & 12,9 & 30,6 & 44,8 & 4,09 & 389 \\
\hline $\begin{array}{l}\text { 3. En casa, vuestro hijo o hija comenta } \\
\text { las tareas realizadas en los ambien- } \\
\text { tes. }\end{array}$ & 3,3 & 13,5 & 16,8 & 30,6 & 35,1 & 3,81 & 396 \\
\hline $\begin{array}{l}\text { 4. Consideráis que vuestro hijo o hija } \\
\text { disfruta de esta manera de trabajar. }\end{array}$ & 0,5 & 1,3 & 10,7 & 27,4 & 59,1 & 4,45 & 390 \\
\hline
\end{tabular}

Fuente: elaboración propia.

Según los datos obtenidos, las familias valoran positivamente la implementación de esta nueva metodología de trabajo, siendo que un 50,6 \% la considera interesante y a un $44,8 \%$ les gustaría también que continuara en la etapa de educación primaria. Es interesante destacar que casi un 60\% de los padres valoran que los hijos aprendan de manera lúdica y placentera.

Tabla 6. Aspectos procedimentales y actitudinales

\begin{tabular}{lccccccccc}
\hline & \multicolumn{7}{c}{ Nivel de acuerdo (\%) } & Media & $\boldsymbol{n}$ \\
\cline { 2 - 6 } & $\mathbf{1}$ & $\mathbf{2}$ & $\mathbf{3}$ & $\mathbf{4}$ & $\mathbf{5}$ & & \\
\hline $\begin{array}{l}\text { 2. Posibilita la relación y la convivencia } \\
\text { con menores de diferentes edades. }\end{array}$ & - & 2,3 & 25,1 & 33,2 & 39,4 & 4,10 & 259 \\
$\begin{array}{l}\text { 3. Favorece una manera de aprender } \\
\text { más atractiva. }\end{array}$ & - & 1,6 & 12,7 & 28,7 & 57 & 4,41 & 251 \\
\hline $\begin{array}{l}\text { 4. Favorece el aprendizaje de la gestión } \\
\text { del tiempo. }\end{array}$ & 0,8 & 3,1 & 22 & 41,7 & 32,3 & 4,02 & 127 \\
$\begin{array}{l}\text { 5. Favorece la motivación y las ganas de } \\
\text { aprender. }\end{array}$ & - & 0,7 & 17,3 & 35,8 & 46,1 & 4,27 & 271 \\
\hline $\begin{array}{l}\text { 6. Permite la estimulación de la creati- } \\
\text { vidad. }\end{array}$ & 0,4 & 0,8 & 23,5 & 34,1 & 40,9 & 4,16 & 264 \\
\hline $\begin{array}{l}\text { 7. Da posibilidades de experimentación } \\
\text { y juego. }\end{array}$ & - & 0,6 & 12,9 & 30,9 & 55,7 & 4,42 & 350 \\
\hline
\end{tabular}

Fuente: elaboración propia.

Los resultados muestran que los padres valoran positivamente los aspectos actitudinales y procedimentales que aporta la nueva metodología: ofrece una manera de aprender más atractiva (un 57\%), posibilita un aprendizaje más experiencial y lúdico (un 55,7\%), fomenta la autonomía (un $47,5 \%$ ) y favorece la motivación hacia el aprendizaje (un 46,1\%). 
Tabla 7. Aspectos conceptuales

\begin{tabular}{lcccccccc}
\hline & \multicolumn{4}{c}{ Nivel de acuerdo (\%) } & \multirow{2}{*}{ Media } & \multirow{n}{*}{} \\
\cline { 2 - 7 } & $\mathbf{1}$ & $\mathbf{2}$ & $\mathbf{3}$ & $\mathbf{4}$ & $\mathbf{5}$ & & \\
\hline 2. Lenguaje y matemáticas. & 0,5 & 2,3 & 29,5 & 35,5 & 32,3 & 3,97 & 220 \\
\hline 3. Expresión artística. & - & 0,9 & 21,3 & 37 & 40,8 & 4,18 & 211 \\
\hline 4. Conocimiento del medio. & - & 2 & 36 & 30,8 & 31,2 & 3,91 & 247 \\
\hline
\end{tabular}

Fuente: elaboración propia.

En cuanto a la valoración acerca de los aspectos conceptuales y curriculares que permite esta metodología, las familias destacan que el desarrollo cognitivo (un 53,1\%) y la expresión artística (un 40,8\%) se favorecen fundamentalmente con este estilo de trabajo.

Cabe destacar cómo las dimensiones de conocimiento y valoración obtienen puntuaciones más altas respecto a la dimensión de participación, considerando así que las familias afirman disponer de información y a partir de ella llevar a cabo una valoración en positivo, pero sin que ello conlleve una participación real.

Finalmente, hemos recogido cuales han sido las dificultades que las familias detectan. Como se puede observar en la tabla 8, parece ser que lo que más les preocupa es el hecho de que se pueda crear confusión en los niños y dudan sobre los resultados de aprendizaje.

Tabla 8. Inconvenientes en la utilización de esta metodología

\begin{tabular}{lcc}
\hline ¿Cuál es el principal inconveniente o duda? & $\boldsymbol{n}$ & $\%$ \\
\hline Crea confusión en los menores. & 112 & 51,1 \\
\hline Los aprendizajes que se realizan son poco relevantes. & 37 & 16,9 \\
\hline No se ven los resultados de los aprendizajes. & 70 & 32 \\
\hline
\end{tabular}

Fuente: elaboración propia.

\section{Discusión y conclusiones}

En relación con la categoría de conocimiento, en los resultados obtenidos las familias manifiestan que están al corriente de la organización y la implementación de esta nueva metodología, aunque dicha información puede no ser suficiente para entender su valor educativo, ya que algunas respuestas señalan que les falta conocimiento acerca de los beneficios que supone esta metodología en el desarrollo y el aprendizaje de sus hijos.

El estudio muestra que la participación de las familias en el proceso de cambio se reduce principalmente a la aportación de material cuando la escuela se lo requiere. Prácticamente no se establecen canales de participación periódica de los padres en comisiones de trabajo o en la observación puntual del funcionamiento de los ambientes, muy lejos queda también la participación 
en la toma de decisiones. La información que han recibido las familias acerca del proceso de cambio ha sido fundamentalmente a través de las primeras entrevistas o reuniones con el tutor y/o con el equipo directivo. Sin duda, diversificar los canales y los escenarios de relación con las familias más allá de la comunicación oral debería ser uno de los objetivos a plantear, dado que la construcción de la relación entre la escuela y las familias pasa previamente por ofrecer informaciones a través de canales que inviten a la participación. Se desprende también la importancia de generar una documentación pedagógica que transmita a los padres y madres de modo sencillo y didáctico la línea educativa y la metodología de trabajo, ya que la experiencia nos ha mostrado que apenas se cuenta con visibilización de los cambios a través de paneles, webs, etc. Como señala Bonás (2012), la documentación permite establecer un diálogo con las familias y profundizar en el sentido de comunidad.

El reto está en proporcionar oportunidades para la participación, mejorar la comunicación y pasar de la implicación al compromiso (Baker et al., 2016). El paso de la participación al compromiso comporta un cambio de actitud de la escuela hacia los padres (Ferlazzo, 2011) y existe todavía cierta tendencia a infravalorar la participación parental en las escuelas por la incertidumbre que supone su inclusión en el proceso de enseñanza-aprendizaje, aunque la finalidad debería ser construir contextos de sintonía que favorecieran los procesos educativos a partir del correconocimiento y la co-acción (Gifre y Guitart, 2012).

Respecto a la consideración de las familias hacia esta nueva metodología es interesante señalar la valoración de aspectos relacionados con la motivación hacia el aprendizaje, la autonomía y la creatividad. Así, han considerado que esta técnica ayuda a facilitar el desarrollo socioemocional y estimula el aprendizaje, pero también reconocen el valor que tiene en relación con el desarrollo cognitivo. Se podría interpretar que los padres parecen conscientes de que no puede haber adquisición de conocimiento sin emociones positivas, por lo que aprecian el valor del juego y de un aprendizaje más experiencial y activo. En este sentido, el informe de la UNESCO (2015) hace hincapié en esta vinculación entre emoción, motivación y cognición.

Es necesario un planteamiento holístico de la educación y del aprendizaje que supere las dicotomías tradicionales entre los aspectos cognitivos, emocionales y éticos. Aumenta el reconocimiento de que superar la dicotomía entre las formas cognoscitivas y otras formas de aprendizaje es esencial para la educación. (UNESCO, 2015, p. 39)

Desde el punto de vista de la investigación realizada, se estiman necesarios estudios en profundidad sobre prácticas y percepciones de la participación parental en los procesos de cambio e innovación educativa, como podría ser analizar los efectos que ejerce en el éxito escolar o estudiar la relación entre participación familiar y mejora de la comunicación con los docentes. Sería necesario incorporar, en trabajos posteriores, el análisis de las causas y las consecuencias de las conclusiones establecidas a partir de los datos recogidos. Este análisis se debería centrar en la necesaria convivencia en el centro educa- 
tivo en el cual se creen lazos de confianza para establecer una base de trabajo conjunto con las familias (Muntaner et al., 2020). Solo así el conocimiento, la participación y la valoración podrán convertirse en realidad. También sería interesante evaluar las consecuencias en el proceso de innovación de la participación real de las familias, como han ido recogiendo diferentes estudios (Osoro y Castro, 2017; Mehlecke, Bernárdez y Belmonte, 2020).

La investigación realizada nos permite afirmar que todavía se está lejos de conseguir que las familias se sitúen en un nivel alto de participación más decisoria que consultiva (Kidder, 2013; Grañeras et al., 2011) y que sería conveniente mejorar el conocimiento parental en relación con sus derechos individuales y colectivos de participación para favorecer la calidad educativa. Cada centro debería trabajar para encontrar la forma de incluir a las familias en los procesos de innovación sin que los docentes sintieran invadidas sus competencias profesionales. Carbonell (2006) habla de impulsar la cultura de la innovación haciendo incidencia en todas las partes involucradas en el cambio y refuerza la idea de que el cambio debe ser consciente y deseado por parte de toda la comunidad educativa.

\section{Referencias bibliográficas}

ANDrÉs, S. y Giró, J. (2016). La participación de las familias en la escuela: Una cuestión compleja. Revista de Evaluación de Programas y Politicas Públicas, 7, 28-47.

Baker, T.L.; Wise, J.; Kelley, G. y Skiba, R.J. (2016). Identifying barriers: Creating solutions to improve family engagement. School Community Journal, 26(2), 161-184. Recuperado de <https://www.adi.org/journal/2016fw/BakerEtAlFall 2016.pdf>.

Barton, A.C.; Drake, C.; Perez, J.G.; Louis, K.S. y George, M. (2004). Ecologies of parental engagement in urban education. Educational Researcher, 33(4), 3-12.

Bonśs, M. (2012). Documentar, una mirada nueva: Red territorial de Educación Infantil de Cataluña. Temas de Infancia, 29. Barcelona: Octaedro.

Carbonell, J. (2006). La aventura de innovar: El cambio en la escuela. Madrid: Morata.

Castro, M.; Expósito, E.; Lizasoain, L.; López, E. y Navarro, E. (2014). Participación familiar y rendimiento académico de alumnos españoles de Educación Infantil, Educación Primaria y Educación Secundaria Obligatoria. En Consejo Escolar del Estado. La participación de las familias en la educación escolar (pp. 167-179). Madrid: Ministerio de Educación, Cultura y Deporte.

Comas, M.; Abellán, C.; Alcantud, A. y Escapa, S. (2014). Llibre Blanc de la participació de les famílies a l'escola [versión electrónica]. Barcelona: Fundació Bofill. Informes Breus, 51.

Comas, M. (dir.); Escapa, S. y Abellán, C. (2014). Com participen mares i pares a l'escola?: Diversitat familiar i d'implicació en educació [versión electrónica]. Barcelona: Fundació Bofill. Informes Breus, 49.

Crozier, G. y Davies, J. (2007). Hard to Reach Parents or Hard to Reach Schools?: A Discussion of Home-School Relations, with Particular Reference to Bangla- 
deshi and Pakistani Parents. British Educational Research Journal, 33(3), 295313.

Duarte, D. (2003). Ambientes de aprendizaje: Una aproximación conceptual. Estudios Pedagógicos (Valdivia), 29, 97-113.

Epstein, J.L. (2001). School and family partnerships: Preparing educators and improving schools. Boulder, CO: Westview Press.

Feito, R. (2010). Familias y escuela: Las razones de un desencuentro. Educación y Futuro: Revista de Investigación Aplicada y Experiencias Educativas, 22, 87-108.

Ferlazzo, L. (2011). Involvement or Engagement? Educational Leadership, 68(8), 10-14.

Fullan, M. (2007). Las fuerzas del cambio con creces. Madrid. Akal.

Garreta, J. (2015). La comunicación familia-escuela en Educación Infantil y Primaria. Revista de la Asociación de Sociología de la Educación, 8(1), 71-85.

- (2017) (coord.). Familias y escuelas: Discursos y prácticas sobre la participación en la escuela. Madrid: Pirámide.

Gifre, M. y Guitart, M.E. (2012). Consideraciones educativas de la perspectiva ecológica de Urie Bronfenbrenner: Contextos educativos. Revista de Educación, $15,79-92$.

Giró, J. y ANDrés, S. (2016). Instalados en la queja: El profesorado ante la participación de las familias en las escuelas. RASE: Revista de la Asociación de Sociología de la Educación, 9(3), 334-345.

Graham-Clay, S. (2005). Communicating with parents: Strategies for teachers. School Community Journal, 15(1), 117-129.

Grañeras, M.; Gil, N. y Díaz-Caneja, P. (2011). Actuaciones de éxito en las escuelas europeas. Madrid: Ministerio de Educación. IFIE. Estudios CREADE, 9.

Grundy, S. (1997). Producto o praxis del curriculum. Madrid: Morata.

Guardia, R.M. de la y Santana, F. (2010). Alternativas de mejora de la participación educativa de las familias como instrumento para la calidad educativa. Revista Iberoamericana sobre Calidad, Eficacia y Cambio en Educación, 8(3), 6-30.

Heath, D.; Maghrabi, R. y Carr, N. (2015). Implications of Information and Communication Technologies (ICT) for School-Home Communication. Journal of Information Technology Education, 14, 363-396.

Hill, N.E. y Craft, S.A. (2003). Parent-school involvement and school performance: Mediated pathways among socioeconomically comparable African American and Euro-American families. Journal of Educational Psychology, 95(1), 74-83.

Hoover-Dempsey, K. (2011). Self-efficacy: Up to the challenge. En S. Redding, M. Murphy y P. SHeley (eds.). Handbook on Family and Community Engagement (pp. 61-68). Lincoln, IL: Academic Development Institute.

KidDer, A. (2013). El paper de les famílies en la millora de l'escola i del sistema educatiu [versión electrónica]. Barcelona: Fundació Bofill. Debats d'Educació, 33.

Kolв, D.A. (2014). Experiential learning: Experience as the source of learning and development. $2 \mathrm{a}$ ed. Nueva Jersey: Pearson Education.

Lee, J.S. y Bowen, N.K. (2006). Parent involvement, cultural capital, and the achievement gap among elementary school children. American Educational Research Journal, 43(2), 193-218.

Lewis, L.L.; KIM, Y.A. y BeY, J.A. (2011). Teaching practices and strategies to involve inner-city parents at home and in the school. Teaching and Teacher Education, 27(1), 221-234. 
LunTs, E. (2003). Parental involvement in children's education: Connecting family and school by using telecommunication technologies. Meridian: A Middle School Computer Technologies Journal, 6(1), 1-8.

Marcelo, C.; Mayor, C. y Gallego, B. (2010). Innovación educativa en España desde el punto de vista de sus protagonistas. Profesorado: Revista de Currículum y Formación del Profesorado, 14(1), 111-134.

Martínez González, R.A. y San Fabián, J.L. (2002). Autoevaluación de la cultura participativa del centro. Organización y Gestión Educativa, 4(85), 21-28.

Mehlecke, Q.T.C.; Bernárdez-Gómez, A. y Belmonte, M.L. (2020). La relación familia-escuela como escenario de colaboración en la comunidad educativa. Revista Valore, 5, 5025.

Muntaner, J.J.; Ferrà, J.; Egea, M.; Zanoguera, M.; Valenzuela, L. y Xaudiera, E. (2020). L'aposta per un canvi global al CEIP Puig de na Fàtima. Bones Pràctiques en Entorns Pedagògics, 6. Recuperado de <https://bones-practiques-pedago giques.uib.es/a/deaf14b1279215681acaffcd531770cd254ec241/numero_6.pdf>.

Murillo, F.J y KRICHesky, G. (2012). El proceso de cambio escolar: Una guía para impulsar y sostener la mejora de las escuelas. Revista Iberoamericana sobre Calidad, Eficacia y Cambio en Educación, 10(1).

Nye, C.; Turner, H. y Schwartz, J. (2006). Approaches to parent involvement for improving the academic performance of elementary school age children. Oslo: Campbell Collaboration.

Osoro, J.M. y Castro, A. (2017). Educación y democracia: La escuela como entorno de participación. Revista Iberoamericana de Educación, 75(2), 89-108.

Otálora, Y. (2010). Diseño de espacios educativos significativos para el desarrollo de competencias en la infancia. Revista CS, 5, 71-96. <https://doi.org/10.18046/recs.i5.452>

Patel, S.; Corter, C. y Pelletier, J. (2008). What do Families Want?: Understanding their goals for early childhood services. En M. Cornish (ed.). Promising Practices for Partnering with Families in the Early Years: Family School Community Partnership Monograph series (pp. 103-135). Charlotte, NC: Information Age Publishing.

Pedró, F. (dir.) (2008). El professorat de Catalunya. Barcelona: Editorial Mediterrània i Fundació Bofill. Polítiques, 60.

PÉrez, A. (2012). Educarse en la era digital. Madrid: Morata.

Ramírez, L.N. (2020). Tendencias de la innovación educativa en los contextos sociales: Análisis del mapeo de literatura. Revista Educación, 44(1), 1-18. <https://doi.org/10.15517/revedu.v44i1.33222>

Ribas, C. (2011). Implementación de una nueva metodología por ambientes: Un estudio de caso [memoria de investigación]. Palma de Mallorca. Universidad de las Islas Baleares.

Riera, M.A.; Ferrer, M. y Ribas, C. (2014). La organización del espacio por ambientes de aprendizaje en la Educación Infantil: Significados, antecedentes y reflexiones. Reladei, 3(2), 19-39.

Schecter, S.R. y Sherry, D.L. (2009). Value Added?: Teachers' Investments in and Orientations Toward Parent Involvement in Education. Urban Education, 44(1), 59-87.

Silberman, M.L. (ed.) (2007). The handbook of experiential learning. San Francisco: John Wiley \& Sons.

Smith, A. (2016). Experiential learning. Cheltenham, UK, y Northampton, MA, EUA: Edward Elgar Publishing Limited. 
Stoll, L. y Fink, D. (1999). Para cambiar nuestras escuelas: Reunir eficacia y mejora. Barcelona. Octaedro.

UNESCO (2015). Repensar la educación: ¿Hacia un bien común universal? París: UNESCO.

Vigo, B.; Dieste, B. y Julve, C. (2015). Construcción de la participación de las familias en la escuela: Un estudio etnográfico. Revista Digital de FEAEE-Aragón sobre Organización y Gestión Educativa, 15(5), 23-26.

Zabalza, M.A. (2000). Construyendo el cambio: Perspectivas y propuestas de innovación educativa. Sevilla: Universidad de Sevilla. 